\title{
A snapshot of genetic and epigenetic basis of arrhythmia and heart failure
}

\author{
Junjie Xiao ${ }^{1,2 *}$, Joost P. G. Sluijter ${ }^{3 *}$, Saumya Das ${ }^{4 *}$, Yiqing Yang ${ }^{5 *}$ and Zhongming Shen ${ }^{1,2}$ \\ ${ }^{1}$ Regeneration and Ageing Lab, Experimental Center of Life Sciences and Innovative Drug Research Center, School of Life Science, Shanghai University, \\ Shanghai, China \\ 2 Shanghai Key Laboratory of Bio-Energy Crops, School of Life Science, Shanghai University, Shanghai, China \\ ${ }^{3}$ Division Heart and Lungs, Department of Cardiology, University Medical Center Utrecht, Utrecht, Netherlands \\ ${ }^{4}$ Cardiovascular Research Institute, Beth Israel Deaconess Medical Center, Harvard Medical School, Harvard University, Boston, MA, USA \\ ${ }^{5}$ Department of Cardiology, Shanghai Chest Hospital, Shanghai Jiao Tong University, Shanghai, China \\ *Correspondence: junjiexiao@live; j.sluijter@umcutrecht.nl; sdas@bidmc.harvard.edu; yiqingyang169@sina.com
}

Edited and reviewed by:

Michael E. Symonds, The University of Nottingham, UK

Keywords: genetic variation, epigenetics, arrhythmia, heart failure, microRNAs

The incidence of cardiac arrhythmia and heart failure continues to rapidly increase, leading to a growing epidemic which constitutes a serious financial burden for society (Chugh et al., 2014; Melman et al., 2014). Despite the standard therapeutic strategies to treat adverse cardiac remodeling that precedes the development of arrhythmia and overt heart failure, their prevalence is still increasing and their morbidity and mortality have not been largely improved (Melman et al., 2014; Sardar et al., 2014). Continued and deeper understanding of the molecular mechanisms responsible for arrhythmia and heart failure will help identify novel effective therapies.

In the past few years, research has implicated the contribution of genetic and epigenetic factors to arrhythmia and heart failure. This research hot topic entitled "Genetic and epigenetic basis of arrhythmia and heart failure" provides a snapshot in this area. The 10 selected papers in this special research topic will enhance current understanding of the genetic and epigenetic basis of arrhythmia and heart failure, and also will expand our knowledge on novel interventions.

In this issue, genetic aspects of arrhythmia were described by 3 different groups. Crump and Abbott (2014) provided an update about arrhythmogenic KCNE gene variants and their roles, and also discussed several challenges in this area. Interestingly, Nielsen et al. (2013) showed that the $\mathrm{I}_{\text {to }}$ regulatory subunit Klf15 was not associated with early-onset long atrial fibrillation in their cohort. Besides familial atrial fibrillation, genome wide association studies (GWAS) have identified common variants in the general population for atrial fibrillation. Yao et al. (2014) gave an overview on the paired-like homeodomain 2, which was identified as a common variant to be involved in atrial fibrillation, highlighting the potential of paired-like homeodomain 2 as a new therapeutic target.

Duygu et al. (2013) provided an outstanding overview about the genetics and epigenetics of arrhythmia and heart failure. Besides highlighting specific genetic mutations associated with heart failure, they also summarized epigenetic mechanisms including DNA methylation, ATP-dependent chromatin remodeling, histone modification and RNA-based mechanisms, highlighting the relationship among epigenetics, heart failure and arrhythmiaogenesis. Finally, they also introduced a discussion on the role of non-coding RNAs especially microRNAs (miRNAs, miRs) in heart failure and arrhythmia.

miRNAs are endogenous non-coding RNAs which posttranscriptionally regulate gene expressions (Condorelli et al., 2014). A single miRNA can target several target genes while an individual gene can be regulated by many miRNAs (Viereck et al., 2014). Therefore, miRNAs have been considered as central regulators of gene expression, which participate in many essential biological processes including cellular proliferation, differentiation, apoptosis, and cardiac metabolism (Condorelli et al., 2014; Viereck et al., 2014). Dysregulations of miRNAs have been connected to many cardiovascular diseases, including arrhythmia and heart failure (Kwekkeboom et al., 2014; Melman et al., 2014). In this special research topic, 4 opinion articles have been provided about miRNAs in arrhythmia and heart failure. Fu et al. (2014) presented the current understanding about miRNAs as novel players in atrial fibrillation, a most common form of arrhythmia that affects at least $1 \%$ persons in the general population. Changes in cardiac structure and function (termed diabetic cardiomyopathy) may play a role in the increased incidence of sudden death and heart failure in the diabetic patients. Zhou et al. (2014) summarized dysregulations of miRNAs in diabetic cardiomyopathy. Interestingly, changes of circulating miRNAs in diabetic patients have also been noted, although their associations with diabetic cardiomyopathy are unclear. Better understanding of circulating miRNAs in diabetic cardiomyopathy would help the development of biomarkers and novel therapies. Ageing is correlated with an increase in the incidence of both heart failure (particularly heart failure with preserved ejection fraction) and atrial fibrillation. Considering the rapid increase in the geriatric population, it is urgent to understand the role of miRNAs in aging. Zhuo et al. (2014) provided some insights into the dysregulation of miRNAs in aging-related heart failure. New therapeutics via targeting microRNAs are being developed based on the detailed understanding of the role of miRNAs in heart failure and arrhythmia using either antagomirs or mimics (Kwekkeboom et al., 2014). In contrast to the previous articles on the role of miRNAs in pathological heart diseases, Fu et al. (2013) dissected the role of 
miRNAs in physiological hypertrophy. Physiological hypertrophy, which is associated with exercise and pregnancy, is not associated with pathological processes like apoptosis and fibrosis, and unlike its counterpart (pathological hypertrophy), is not the harbinger of sudden death or heart failure. Given the known beneficial effects of exercise on cardiovascular outcomes, a better understanding of miRNAs in physiological hypertrophy may lead to novel miRNA-based therapies for heart failure.

Finally Stagnaro and Caramel (2013) proposed a "Quantum Biophysics Semeiotics" (QBS) microcirculatory theory of atherosclerosis, which could be used for diagnosis or therapies. In addition, Tao et al. (2013) provided their prospects of using Qiliqiangxin, a traditional Chinese medicine, in the treatment of heart failure potentially through epigenetic regulation of regeneration.

In conclusion, this research topic offers a detailed and updated summary about genetic and epigenetic basis of arrhythmia and heart failure, and provides a forum for discussion on ways to translate these genetic and epigenetic findings into novel therapies for arrhythmia and heart failure.

\section{REFERENCES}

Chugh, S. S., Roth, G. A., Gillum, R. F., and Mensah, G. A. (2014). Global burden of atrial fibrillation in developed and developing nations. Glob. Heart 9, 113-119. doi: 10.1016/j.gheart.2014.01.004

Condorelli, G., Latronico, M. V., and Cavarretta, E. (2014). microRNAs in cardiovascular diseases: current knowledge and the road ahead. J. Am. Coll Cardiol. 63, 2177-2187. doi: 10.1016/j.jacc.2014.01.050

Crump, S. M., and Abbott, G. W. (2014). Arrhythmogenic KCNE gene variants: current knowledge and future challenges. Front. Genet. 5:3. doi: 10.3389/fgene.2014.00003

Duygu, B., Poels, E. M., and da Costa Martins, P. A. (2013). Genetics and epigenetics of arrhythmia and heart failure. Front. Genet. 4:219. doi: 10.3389/fgene.2013.00219

Fu, S., Huang, L., Wang, Y., Li, X., Li, J., and Xiao, J. (2014). MicroRNA as a novel player in atrial fibrillation. Front. Genet. 5:97. doi: 10.3389/fgene.2014.00097

Fu, S., Zhuo, R., Yao, M., Zhang, J., Zhou, H., and Xiao, J. (2013). MicroRNA basis of physiological hypertrophy. Front. Genet. 4:253. doi: 10.3389/fgene.2013.00253

Kwekkeboom, R. F., Lei, Z., Doevendans, P. A., Musters, R. J., and Sluijter, J. P. (2014). Targeted delivery of miRNA therapeutics for cardiovascular diseases: opportunities and challenges. Clin. Sci. (Lond.) 127, 351-365. doi: 10.1042/CS20140005

Melman, Y. F., Shah, R., and Das, S. (2014). MicroRNAs in heart failure: is the picture becoming less miRky? Circ. Heart Fail. 7, 203-214. doi: 10.1161/CIRCHEARTFAILURE.113.000266

Nielsen, M. W., Olesen, M. S., Refsgaard, L., Haunsø, S., and Svendsen, J. H. (2013). Screening of the ito regulatory subunit klf15 in patients with early-onset lone atrial fibrillation. Front. Genet. 4:88. doi: 10.3389/fgene.2013.00088

Sardar, M. R., Saeed, W., and Kowey, P. R. (2014). Antiarrhythmic drug therapy for atrial fibrillation. Cardiol. Clin. 32, 533-549. doi: 10.1016/j.ccl.2014.07.012

Stagnaro, S., and Caramel, S. (2013). The key role of vasa vasorum inherited remodeling in QBS microcirculatory theory of atherosclerosis. Front. Genet. 4:55. doi: 10.3389/fgene.2013.00055

Tao, L., Shen, S., and Li, X. (2013). Future prospects of Qiliqiangxin on heart failure: epigenetic regulation of regeneration. Front. Genet. 4:221. doi: 10.3389/fgene.2013.00221

Viereck, J., Bang, C., Foinquinos, A., and Thum, T. (2014). Regulatory RNAs and paracrine networks in the heart. Cardiovasc. Res. 102, 290-301. doi: $10.1093 / \mathrm{cvr} / \mathrm{cvu} 039$

Yao, M., Cao, Y., Zhu, H., Chen, Y., Zhu, T., and Xiao, J. (2014).Paired-like homeodomain 2: a novel therapeutic target for atrial fibrillation? Front. Genet. 5:74. doi: 10.3389/fgene.2014.00074

Zhou, Q., Lv, D., Chen, P., Xu, T., Fu, S., Li, J., et al. (2014). MicroRNAs in diabetic cardiomyopathy and clinical perspectives. Front. Genet. 5:185. doi: 10.3389/fgene.2014.00185

Zhuo, R., Fu, S., Li, S., Yao, M., Lv, D., Xu, T., et al. (2014). Desregulated microRNAs in aging-related heart failure. Front. Genet. 5:186. doi: 10.3389/fgene.2014.00186

Conflict of Interest Statement: The authors declare that the research was conducted in the absence of any commercial or financial relationships that could be construed as a potential conflict of interest.

Received: 29 January 2015; accepted: 12 February 2015; published online: 03 March 2015

Citation: Xiao J, Sluijter JPG, Das S, Yang Y and Shen Z (2015) A snapshot of genetic and epigenetic basis of arrhythmia and heart failure. Front. Genet. 6:74. doi: 10.3389/ fgene.2015.00074

This article was submitted to Epigenomics and Epigenetics, a section of the journal Frontiers in Genetics.

Copyright (c) 2015 Xiao, Sluijter, Das, Yang and Shen. This is an open-access article distributed under the terms of the Creative Commons Attribution License (CC BY). The use, distribution or reproduction in other forums is permitted, provided the original author(s) or licensor are credited and that the original publication in this journal is cited, in accordance with accepted academic practice. No use, distribution or reproduction is permitted which does not comply with these terms. 